\title{
An Effective Way to Process Linear Frequency Modulation Signal
}

\section{Liangwei Feng1 and Jun Chen1, Hao Wu2 and Feiyun Guan2}

School of Aeronautics and Astronautics of University of Electronic Science and Technology of China, Chendu 611731, China;

195241163@qq.com , 2050305649@qq.com,1156299831@qq.com , guanfeiyunhotmail@ccc.com

Keywords: Rearrangement of the Smooth Pseudo Wigner-Ville Distribution (RSPWVD), Linear Frequency Modulation (LFM) Signal, Modified Fast Hough Transform (MFHT).

Abstract. WVD is most commonly used to do Signal processing in the time-frequency domain. Hough transform is a commonly used and classic way to do line detection. But in the field of engineering, there are higher demands for the speed of signal processing. Therefore, we could apply the Fast Hough Transform algorithm in our projects.

\section{Introduction}

This document presents an effective way to get better results of processing LFM (Linear Frequency Modulation) signal by using a combination of Rearrangement of the Smooth Pseudo Wigner-Ville Distribution (RSPWVD) and a Modified Fast Hough Transform.

\section{Interference existed when processing FLM signal}

LFM Signal is a typical non-stationary signal which has high resolution. However, The cross term often exists when many methods are used to process signals in the time-frequency domain, especially for WVD. As a matter of fact, the first problem is how to extract the key parameters of signal when we get a LFM signal because there is a lot of noise. And the process of removing noise is also a helpful way to extract some key parameters of the LFM signal. So, a good way of dealing with this problem will be introduced in detail next.

\section{Candidate Algorithms to Process FLM signal}

\subsection{Introduction of RSPWVD Distribution}

If $z(n)$ is discrete analytic signal and the signal length is $2 L+1$, Let $W_{z}(h, k)$ represent discrete WVD. The mathematical expression is given below.

$$
W_{z}(n, k)=2 \sum_{m=-L}^{L} z(n+m) z^{*}(n-m) e^{-j 4 \pi k m / N}
$$

Many non-stationary signals have multiple component signals with complex time-frequency characteristics which means those component signals may have cross term together. Therefore, we could use RSPWVD to process FLM signal which can reduce the interference of cross term and improve the resolution of results of time-frequency analysis. The mathematical expression of RSPWVD is given below.

Where

$$
R S P W_{x}(t, \omega)=\int_{-\infty}^{+\infty} \int_{-\infty}^{+\infty} S P W_{x}\left(t^{\prime}, \omega^{\prime}\right) \delta\left(t-\hat{t}\left(x ; t^{\prime}, \omega^{\prime}\right)\right) \delta\left(\omega^{\prime}-\hat{\omega}\left(x ; t^{\prime}, \omega^{\prime}\right)\right) d t^{\prime} d \omega^{\prime}
$$

$$
\hat{t}(x ; t, \omega)=\frac{\iint \tau L(\tau, \theta) S P W_{x}(t-\tau, \omega-\theta) d \tau d \theta}{\iint L(\tau, \theta) S P W_{x}(t-\tau, \omega-\theta) d \tau d \theta}
$$




$$
\hat{\omega}(x ; t, \omega)=\frac{\iint \theta L(\tau, \theta) S P W_{x}(t-\tau, \omega-\theta) d \tau d \theta}{\iint L(\tau, \theta) S P W_{x}(t-\tau, \omega-\theta) d \tau d \theta}
$$

\subsection{Introduction of a Modified Fast Hough Transform Algorithm}

Fast Hough Transform achieves rapid calculation through approximation. There are basic thoughts below to implement the FHT algorithm.

(1). $\mathrm{N}$ represents the image size , $N=2^{k}$. If $\neq 2^{k}$, using zero fill in $x$ direction and $y$ direction to make $N=2^{k}$.

(2). During initialization, let the size of original image extend to $2 N * N$ by using zero fill in $\mathrm{x}$ direction which means let $\mathrm{g}\left(x_{i}, y_{i}\right)=\mathrm{g}\left(x_{i+2 N}, y_{i}\right)$

(3). In step $i$ of iterative processing, the line to be calculated should have characteristics as below.

i. $\quad x=0,1,2, \cdots, 2 N-1$ where $x$ is the $x$ coordinate of the line;

$$
\begin{aligned}
& y_{\text {start }}=j \cdot 2^{i} ; \quad y_{\text {end }}=(j+i) \cdot 2^{i}-1, \\
& j=0,1, L\left(\frac{N}{2^{i}}\right)-1
\end{aligned}
$$

ii. Where $y_{\text {start }}$ is the $y$ coordinate of the start of the line, $y_{\text {end }}$ is the $y$ coordinate of the end of the line .

$$
\operatorname{tg} \theta=\frac{a}{2^{i}-1}, \quad a=0,1, \mathrm{~L}, 2^{i}-1
$$

,where $\theta$ is the inclination of the line.

iii.

$$
H_{i}(x, y, a)=H_{i-1}\left(x, y,\left[\frac{a}{2}\right]\right)+H_{i-1}\left(x-\left[\frac{a}{2}\right], y+2^{i-1},\left[\frac{a}{2}\right]\right)
$$

where $H_{i}(x, y, a)$ is the value of the line calculated in step $i$.

The Modified Fast Hough Transform is based on the idea of "Expanding-FHT-Extraction". Although the amount of calculation is more comparing with FHT, the MFHT could reduce errors effectively and improve the computing accuracy. The MFHT algorithm is given as below.

1. Expanding

$$
\hat{g}(\hat{x}, \hat{y})= \begin{cases}g\left(\left[\frac{x}{k}+\frac{1}{2}\right],\left[\frac{y}{k}\right]\right), & y \equiv 0(\bmod k) \\ 0, & \text { otherwise }\end{cases}
$$

2. FHT

/ * To get data set $\hat{H}_{\log _{2}{ }^{k N}}(\hat{x}, 0, \hat{a})$ by FHT Algorithm $*$ /

3. Contraction

$$
H(x, a)=\hat{H}_{\log _{2}{ }^{k N}}\left(k x, 0,\left(k+\left[\frac{k-1}{N-1}\right]+\frac{1}{2}\right) a\right)
$$

Where $\mathrm{g}(x, y)$ means the original image; $N$ is the size of original image, $g(\hat{x}, \hat{\mathrm{y}})$ means the extended image,; $k N$ is the scale of extension of original image where $k N=k * N$ and $k$ is of integer power; $\widehat{H}_{\log _{2}}{ }^{k N}(\hat{x}, 0, \hat{a})$ is the final computation results of the extended image with FHT algorithm where $\hat{x}=0,1,2, \cdots k N-1$ and $\hat{a}=0,1,2, \cdots k N-1 ; H(x, a)$ is the computation results of MFHT algorithm where $x=0,1, \cdots, 2 N-1$ And $x=0,1, \cdots, N-1$. 


\section{Simulations of LFM signal processing with RSPWVD and MFHT}

As we have seen from the preceding descriptions, we can present our new algorithm to extract the parameters of LFM signal $x(t)$ as follows:

Step 1: execute discrete digitization sampling processing to $x(t)$ to get $x^{\prime}(t)$ which is a sampling vector of $x(t)$.

Step 2: process $x^{\prime}(t)$ with RSPWVD to get time-frequency distribution image of $x(t)$.

Step 3: get peak of each LFM signal by using MFHT to process the time-frequency distribution image, meanwhile getting the key parameters of signal.

Step 4: reconstruct the original time-frequency distribution image of LFM signal by making use of the extracted key parameters. And in this process the noise is also suppressed.

Next we will conduct simulations and prove the method mentioned above is effective and practicable by making comparison between simulation images.

In our simulation, the signal ready to be processed consists of two different LFM signals, where $\tau=10-6 s, B_{1}=300 \mathrm{M}$ and $B_{2}=600 \mathrm{MHz}, f_{0}=0$, the normalized amplitude is between -1 and 1 . From those parameters we could get $\mu_{1}=3 * 10^{14} \mathrm{~Hz} / \mathrm{s}$ and $\mu_{2}=6 * 10^{14} \mathrm{~Hz} / \mathrm{s}$.Fig. 1 shows the ideal time-frequency distribution. The simulation results are illustrated in Fig.2 and Fig.3.

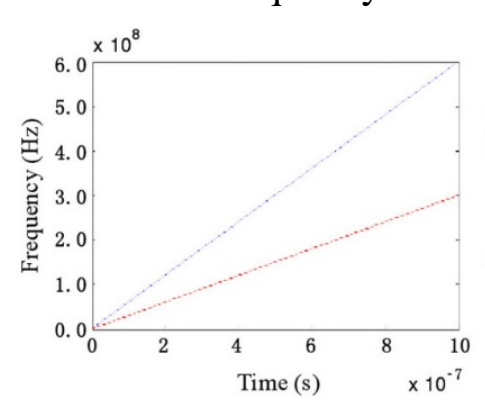

Fig.1 Ideal time-frequency distribution image

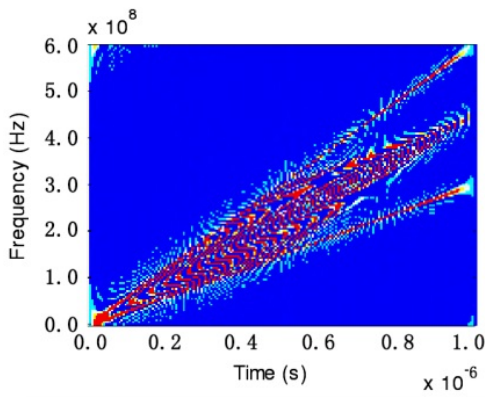

Fig.2 The time-frequency distribution image of signal processed with WVD

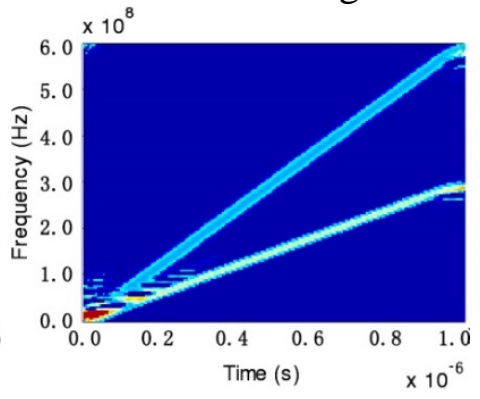

Fig.3 The time-frequency distribution image of signal processed with RSPWVD

Now we will carry out simulation of reducing Gaussian white noise of LFM signal, extracting the parameters of LFM signal at the same time. The simulation results are shown in Fig.4 and Fig.5, where $\mathrm{SNR}=-5 d B$.

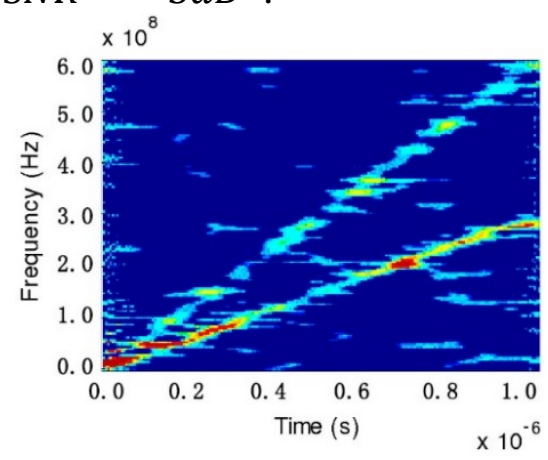

Fig.4 The time-frequency distribution image of signal processed with RSPWVD

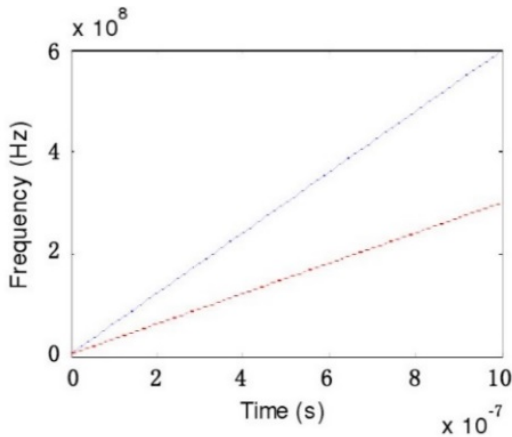

Fig.5 The reconstructed time-frequency distribution image with MFHT

Then Fig.6 and Fig.7 shows the simulation results where $S N R=-10 d B$. 


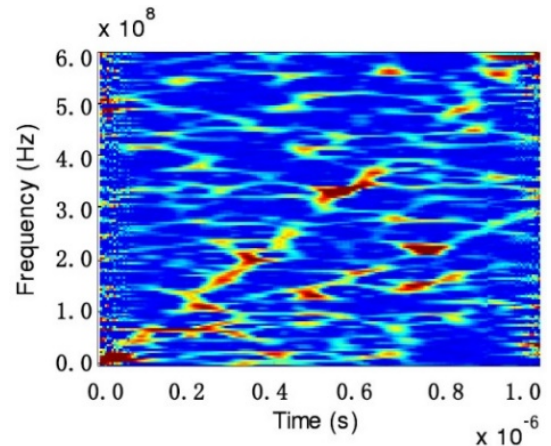

Fig. 6 the time-frequency distribution image of

signal processed with RSPWVD

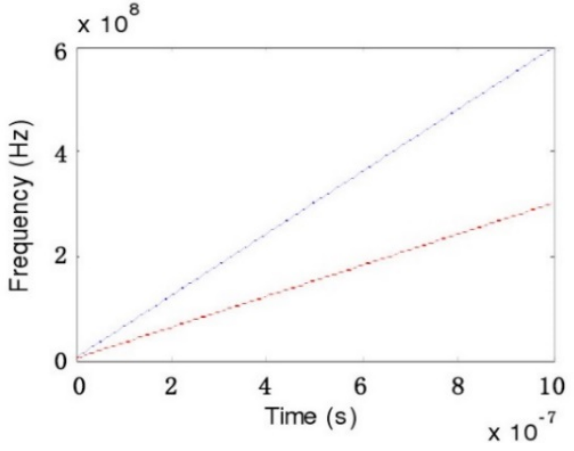

Fig.7 the reconstructed time-frequency distribution image with MFHT

Comparing Fig.7 and Fig.5 with Fig.1, we can say we could get very good results through our methods, where $\mathrm{S} N R>=-10 \mathrm{~dB}$.

Fig. 8 shows the simulation results where $S N R=-13 d B$.Fig.9 and Fig.10 show the change of peak with time of each LFM signal. We can see the peak is smaller and smaller with the SNR decreasing. And our methods are no longer effective when $\mathrm{S} N R<=-13 d B$.

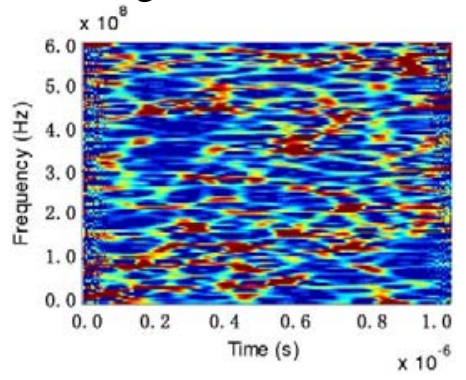

Fig.8 The time-frequency distribution image of signal processed with RSPWVD

Fig.9 Peak Extraction of

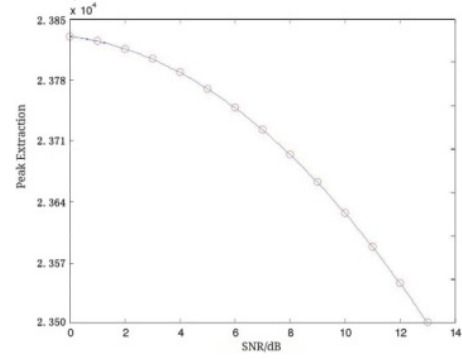

Signal I

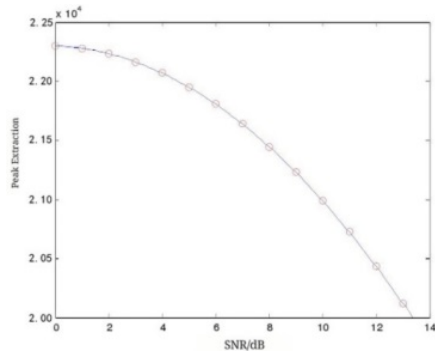

Fig.10 Peak Extraction of Signal II

\section{Conclusions}

Using RSPWVD to process LFM signal could decrease the influence of cross term obviously, also improving the resolution of the results of time and frequency analysis. The MFHT could extract key parameters of each LFM signal effectively. In the process of reconstructing the time-frequency distribution image with extracted parameters, the noise gets removed as well. Our methods can get good results where $\mathrm{S} N R>-10 d B$. But when $\mathrm{S} N R \leq-13 d B$, our methods are no longer effective.

\section{References}

[1]. YM Chee, AZ Sha'ameri .Adaptive windowed cross Wigner-Ville distribution as an optimum phase estimator for PSK signals .Digital Signal Processing, 2013.

[2]. Meng Xie.An improved Hough transform for line detection.Computer Application and System Modeling (ICCASM), 2010 International Conference on (Volume:2).

[3]. Shengzhi Du.An improved Hough transform neighborhood map for straight line segments.Image Processing, IEEE Transactions on (Volume:19 , Issue: 3).

[4]. SHIH-HSUAN CHIU, JIUN-JIAN LIAW, and KUO-HUNG LIN.A FAST RANDOMIZED HOUGH TRANSFORM FOR CIRCLE/CIRCULAR ARC RECOGNITION.Int. J. Patt. Recogn. Artif. Intell. 24, 457 (2010).

[5]. Duan Rujiao,Zhao Wei,Huang Songling,Chen Jianye.Fast line detection algorithm based on 
improved Hough transformation. 《Chinese Journal of Scientific Instrument》,2010-12.

[6]. Research of Suppressing Cross Term of Wigner-ville Distribution Based on EMD. 《Turbine Technology》,2010-03. 